\title{
Ein einfaches Verfahren zum Abschätzen des UV-Index
}

\section{A Simple Procedure for Estimating UV Index}

\author{
Autoren \\ H. Meffert, P. J. Meffert, G. Kolde, E. Rowe \\ Institut \\ Dermatologisches Zentrum Berlin
}

\section{Bibliografie}

DOI $10.1055 / \mathrm{s}-2008-1077624$

Online-Publikation: 8. 10. 2008

Akt Dermatol 2009; 35:

25-28 @ Georg Thieme

Verlag KG Stuttgart · New York ISSN 0340-2541

Korrespondenzadresse

Prof. Dr. med. Hans Meffert

Dermatologisches Zentrum

Potsdamer Chaussee 80

14129 Berlin

hans.meffert@web.de

www.dermatologie-berlin.de

\section{Zusammenfassung \\ $\nabla$}

Beschreibung eines einfachen Verfahrens, mit dem jederzeit und an jedem Ort die aktuell einwirkende solare erythemwirksame UV-Bestrahlungsstärke (UV-Strahlungsfluss) ausreichend genau abgeschätzt werden kann. Es basiert auf der Verknüpfung von UV-Index und Schattenregel und der Anwendung eines Rechenprogramms von O. Engelsen [3]. Ein UV-Messinstrument ist nicht erforderlich. Gemessen werden lediglich die eigene Körpergröße und die Länge des dazugehörigen Schattens. Daraus kann der aktuelle UV-Index leicht errechnet werden.

\section{Einleitung}

\section{$\nabla$}

$\mathrm{Zu}$ viel wie zu wenig Sonnenschein schadet [1]. Der Mensch kann Helligkeit und Dunkelheit, Hitze und Kälte, Nässe und Trockenheit, Lärm und Stille wahrnehmen. Leider verfügt er nicht über ein Sinnesorgan, das ihn über zu viel oder zu wenig ultraviolette Strahlung (UV) informiert. So tritt der Sonnenbrand als Symptom akuter Überdosierung erst Stunden nach übermäßiger UVExposition in Erscheinung. Die Symptomatik chronischer Unterdosierung wird bestenfalls nach Monaten, oft niemals erkannt [2].

Der Mensch ist dem modernen Leben nur teilweise angepasst, das ihn jetzt konfrontiert mit

- überwiegendem Aufenthalt in geschlossenen, verglasten Räumen, oft bei künstlicher Beleuchtung,

- den Gefahren, die infolge der Verminderung atmosphärischen Ozons drohen (vermehrte UV-Penetration zur Erdoberfläche),

- einer UV-B-filternden Smogglocke bei weitgehender Bedeckung der Haut mit Kleidern,

- Migration und Massentourismus in ungewohnte Klimazonen,
Ist der eigene Schatten

- so lang wie man groß ist, so entspricht das dem UV-Index von 4

- um $1 / 3$ länger als man groß ist, so entspricht das dem UV-Index $<3$

Den aktuellen Empfehlungen der WHO und anderer Gremien zufolge sind Schutzmaßnahmen ab UV-Index $=3$ erforderlich. Randbedingungen, Vorzüge und Limitierungen des Verfahrens werden diskutiert. Vergleiche mit gemessenen UVIndices werden vorgenommen.

\section{stellt.}

Wesentliche Voraussetzungen für dessen Entwicklung waren der UV-Index (UVI), die Schattenregel und ein Rechenprogramm von O. Engelsen [3]. Zunächst werden UVI und Schattenregel besprochen. Dann wird beschrieben, wie beide zum Abschätzen der aktuellen Bestrahlungsstärke miteinander verknüpft werden. Mithilfe des so ermittelten UVI ist leicht herauszufinden, wie lange man sich dem Sonnenschein am konkreten Ort und zur konkreten Zeit aussetzen darf. 


\section{Der UV-Index (UVI) \\ $\nabla$}

Der UVI ist ein Maß für die Stärke des Sonnenscheins, genau gesagt für die Erythemwirksamkeit der Sonneneinstrahlung. Er kann Werte von 1 - 10 und mehr erreichen. Dabei stehen kleinere Zahlen für geringere Erythemwirksamkeiten. Um die Mittagszeit ist die Erythemwirksamkeit der Sonnenstrahlung in der Regel am größten. Die Berechnung des UVI erfolgt durch Multiplikation der erythemwirksamen Bestrahlungsstärke $\mathrm{E}_{\mathrm{er}}$ mit der Zahl 40. Für die Referenzsonne nach DIN 67501 [4], die das Spektrum der Sonneneinstrahlung in Meeresspiegelhöhe bei einem Sonnenhöhenwinkel (siehe unten) von $\gamma=90^{\circ}$, bei wolkenlosem und klarem Himmel und bei der für die Tropen vergleichsweise hohen Ozonkonzentration von 320 Dobson-Einheiten beschreibt, beträgt die erythemwirksame Bestrahlungsstärke $E_{e r}=0,2541 \mathrm{~W} / \mathrm{m}^{2}$, was einer sehr starken UV-Einstrahlung der Sonne entspricht. Der zugeordnete UV-Index beläuft sich damit auf $U V I=0,2541 \times 40=10,164$. Nach ganzzahliger Rundung ergibt sich für die Referenzsonne der UVI $=10$.

Für Vorhersagen wird meist der zu erwartende Mittagswert des UVI als ganze Zahl angegeben, der bei gleichbleibender atmosphärischer Transparenz dem Tagesspitzenwert entspricht. Ein prognostizierter UVI von 1 bedeutet, dass an diesem Tage bei Sonnenhöchststand eine erythemwirksame Bestrahlungsstärke von maximal $25 \mathrm{~mW} / \mathrm{m}^{2}$ erwartet wird. Analog entspricht ein UVI von 2 einer erythemwirksamen Bestrahlungsstärke von $50 \mathrm{~mW} / \mathrm{m}^{2}$ usf.

Solche Maximalwerte können kostenlos aus dem Internet entnommen werden, beispielsweise für Deutschland, das hierfür in drei Regionen unterteilt wurde [5]. Als Beispiel zeigt $\bullet$ Abb. 1 den Jahresgang der Tagesspitzenwerte des UVI für das Jahr 2007 im Meteorologischen Observatorium Lindenberg (geografische Breite $52^{\circ} \mathrm{N}$ ), die aus täglichen Messungen im Minutentakt mit Spektralradiometern des Typs Spectro 320 D berechnet wurden. Die UVI sind mit Ampelfarben bewertet. Von Anfang Oktober bis Mitte März bleibt der in Lindenberg gemessene UVI im grünen Bereich ( $\bullet$ Abb. 1).

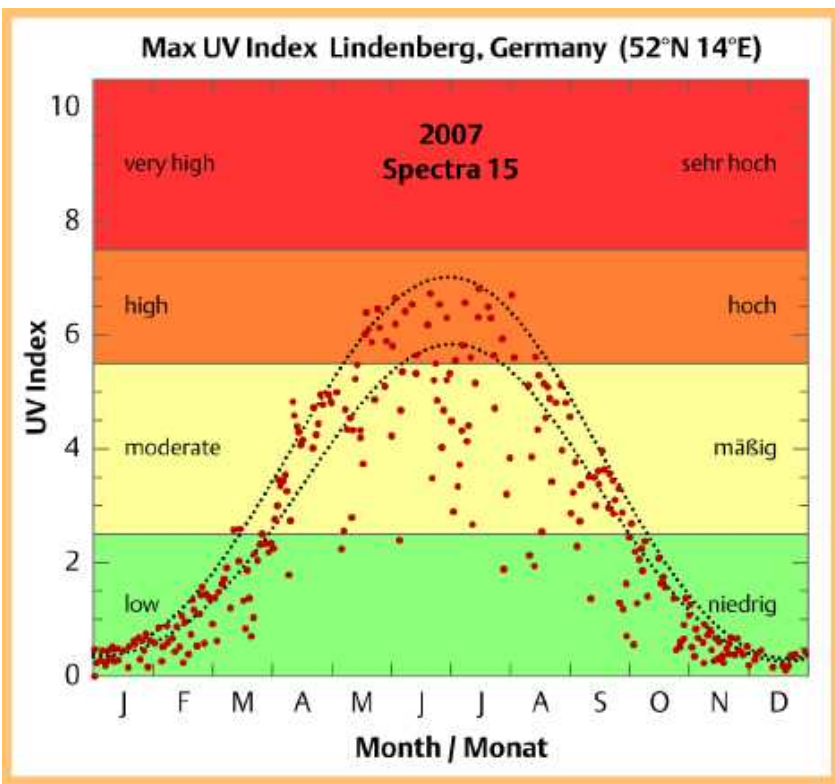

Abb. 1 Jahresgang 2007 des UVI, gemessen im Meteorologischen Observatorium Lindenberg.
Angegeben sind die täglichen Höchstwerte der 30-Minuten-Mittel (rote Punkte). Die für geringe (obere Kurve) und hohe Ozonwerte (untere Kurve) mit einem Strahlungstransportmodell für wolkenlosen Himmel und mittlere Aerosolkonzentration berechneten typischen Jahresgänge des UVI sind durch blaue Punkte dargestellt. Wir danken Herrn Dr. rer. nat. Uwe Feister. Die Weltgesundheitsorganisation (WHO), der Australische Krebsrat (Cancer Council Australia), die Internationale Beleuchtungskommission (CIE), die Internationale Kommission zum Schutz vor nicht-ionisierender Strahlung (ICNIRP), das Bundesamt für Strahlenschutz und andere Gremien empfehlen bei maximalen UVI von

- bis 2,5 - grüner Bereich: kein Schutz erforderlich

- 3 bis 7 - von 11 bis 15 Uhr den Schatten suchen, Schutz durch Kleidung und Creme

- 8 bis 10 - von 11 bis 15 Uhr im Hause bleiben, ansonsten ist in dieser Zeit Schutz durch Kleidung und Creme obligatorisch.

\section{Die Schattenregel \\ $\nabla$}

Je höher die Sonne steigt, umso kürzer werden die Schatten. Der Winkel, der vom Horizont und einem den Kopf einer aufrecht stehenden Person gerade nicht mehr tangierenden Sonnenstrahl gebildet wird, ist der Sonnenhöhenwinkel (siehe $\bullet$ Abb. 2). Er kann maximal $90^{\circ}$ erreichen.

Für Schattenlänge $=1$ ist der UV-Index $=4$.

Für Schattenlänge $=1+1 / 3$ ist der UV-Index $<3$.

Der Physiker L. Holloway hat den mittleren UV-B-Anteil des Sonnenscheins an klaren Sommertagen zu unterschiedlichen Tageszeiten gemessen und in Beziehung zum Sonnenhöhenwinkel und zur Erythemwirksamkeitskurve der Internationalen Beleuchtungskommission (CIE) gesetzt. Es stellte sich heraus, dass bei einem Sonnenhöhenwinkel von $>45^{\circ}$ die Sonnenbrandwahrscheinlichkeit fünfmal größer war als für den Rest des Tages [6]. Wenn dagegen der Schatten eines Menschen vom Hauttyp II deutlich länger war als dessen Körpergröße, so kam es erst nach

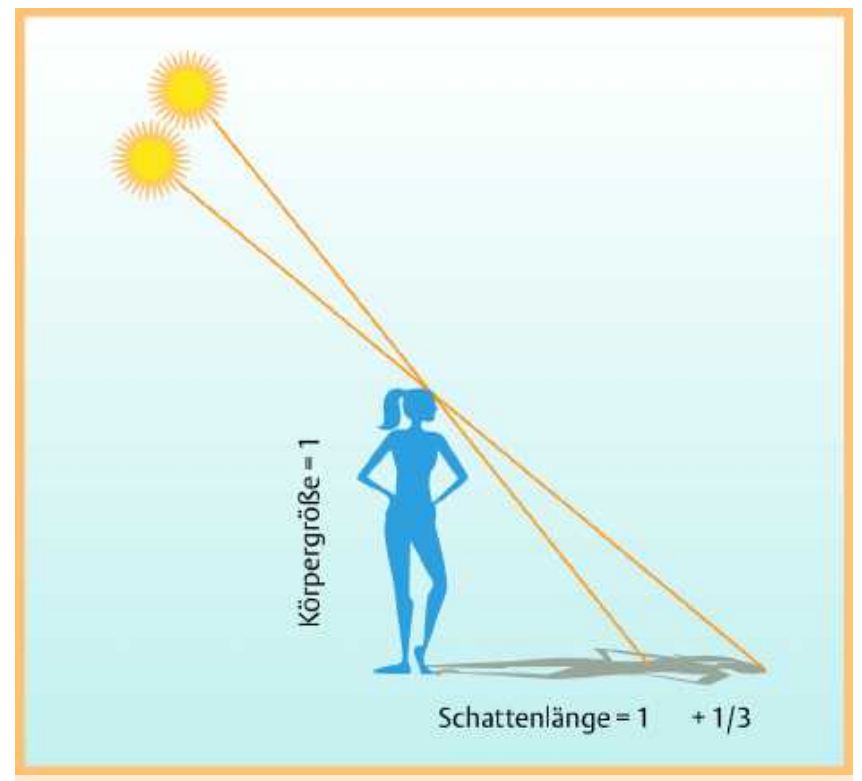

Abb. 2 Der vom Horizont und einem den Kopf der Person tangierenden Sonnenstrahl gebildete Sonnenhöhenwinkel wird mit steigendem Sonnenstand größer. 
mehr als einstündiger Besonnung zum Sonnenbrand [7]. Holloway betonte, dass zum Vermeiden übermäßiger Sonnenexposition einfache und jedermann verständliche Botschaften verbreitet werden sollten [8].

\section{Methode zur nicht-instrumentellen Abschätzung des UV-Index}

Aus dem Internet [5], Presse, Funk und Fernsehen sind prognostizierte Tagesspitzenwerte des UVI ausgewählter Regionen zu erfahren. Gestützt auf Messdaten des Deutschen Wetterdienstes veröffentlicht das Bundesamt für Strahlenschutz dreimal wöchentlich manuell erstellte Dreitagesprognosen der UVI bei Sonnenhöchststand, die jeweils für die Regionen Nord-, Mittel- oder Süddeutschland gelten [5]. Gute Gründe sprechen für eine aktuelle Abschätzung des UVI an Ort und Stelle. Dies kann mithilfe geeigneter Messgeräte geschehen. Dabei sind technische Probleme und unter Umständen verhängnisvolle Bedienungsfehler nicht auszuschließen. Wir entwickelten deshalb ein einfaches und anschauliches Verfahren, das ohne Messgerät auskommt und jederzeit und an jedem Orte angewandt werden kann. Es basiert auf der Schattenregel und erfordert als Messparameter lediglich die eigene Körpergröße und Schattenlänge. Aus diesen zwei leicht messbaren Größen ergibt sich der Sonnenhöhenwinkel $\gamma$ (siehe $\odot$ Abb. 2), dem der UVI proportional ist. Unter Verwendung eines Rechenprogramms von O. Engelsen [3] ergeben sich die in $\odot$ Tab. 1 dargestellten Abhängigkeiten.

Sind Körpergröße und Schattenlänge gleich groß, so entspricht das einem aktuellen UVI von 3,7. Ist der eigene Schatten um $1 / 3$ länger als man selbst groß ist, so ergibt sich ein aktueller UV-Index von $<3$.

In $\odot$ Tab. 1 sind weiterhin die kleinen Standardabweichungen auffällig, die durch jahresperiodische Schwankungen entstehen (vergleiche auch $\bigcirc$ Abb.4). Die angegebenen Werte des UVI können in der Natur, vor allem durch Trübung und Bewölkung, erheblich verkleinert werden.

In $\odot$ Abb. 3 ist der errechnete Tagesspitzenwert des UVI in Abhängigkeit von der Jahreszeit für vier Regionen dargestellt.

Ganz anders als auf Mallorca oder gar am Äquator wird auf Rügen (etwa $54^{\circ} \mathrm{N}$ ) von Anfang Oktober bis Mitte März der UVI $=2,4$ nicht erreicht ( $\odot$ Abb.3). Dieses Ergebnis ähnelt in Dänemark bei $56^{\circ} \mathrm{N}$ durchgeführten Dosimetermessungen [9].

Die aus Körpergröße und Schattenlänge von uns abgeschätzten aktuellen UVI erweisen sich auch im Vergleich zu den in Lindenberg gemessenen Werten als plausibel (vergleiche $\bullet$ Abb. 1,3 und 4).

In $\bigcirc$ Abb. 4 ist die geringe jahresperiodische Schwankung des maximalen UVI dargestellt. Die errechneten Werte können in der Natur vor allem durch Trübung, Bewölkung und Höhe modifiziert werden.

\section{Diskussion}

$\nabla$

Das UV-Strahlenklima der Erde ist eine äußerst komplizierte Materie und kann nur unter Berücksichtigung diverser Randbedingungen exakt beschrieben werden. Die hier vorgestellte Methode zur Abschätzung des aktuellen UV-Index soll jederzeit für jedermann anwendbar sein. Sie ist in diesem Sinne alltagstauglich, weil sie nur wesentlich modifizierende Randbedingungen berücksichtigt. Die Genauigkeit der selbst ermittelten UVI ist

\begin{tabular}{|llll}
\hline \multicolumn{3}{l}{$\begin{array}{l}\text { Tab. } \mathbf{1} \\
\text { Abhängigkeit des UV-Index vom Sonnenhöhenwinkel } \gamma \text {. }\end{array}$} \\
$\begin{array}{l}\text { Körpergröße/ } \\
\begin{array}{l}\text { Schattenlänge } \\
\text { [tan } \gamma \text { ] }\end{array}\end{array}$ & $\begin{array}{l}\text { Sonnenhöhen- } \\
\text { winkel } \gamma\end{array}$ & UVI & $\begin{array}{l}\text { Standard- } \\
\text { abweichung } \\
\text { des UVI }\end{array}$ \\
\hline 4 & 76,0 & 8,5 & 0,2 \\
\hline $\mathbf{2}$ & 63,4 & 6,8 & 0,2 \\
\hline 1,5 & 56,3 & 5,7 & 0,1 \\
\hline $\mathbf{1}$ & $\mathbf{4 5 , 0}$ & $\mathbf{3 , 7}$ & 0,1 \\
\hline $\mathbf{0 , 7 5}$ & $\mathbf{3 6 , 9}$ & $\mathbf{2 , 4}$ & 0,1 \\
\hline 0,5 & 26,6 & 1,2 & 0,03 \\
\hline 0,25 & 14,0 & 0,3 & 0,01 \\
\hline
\end{tabular}

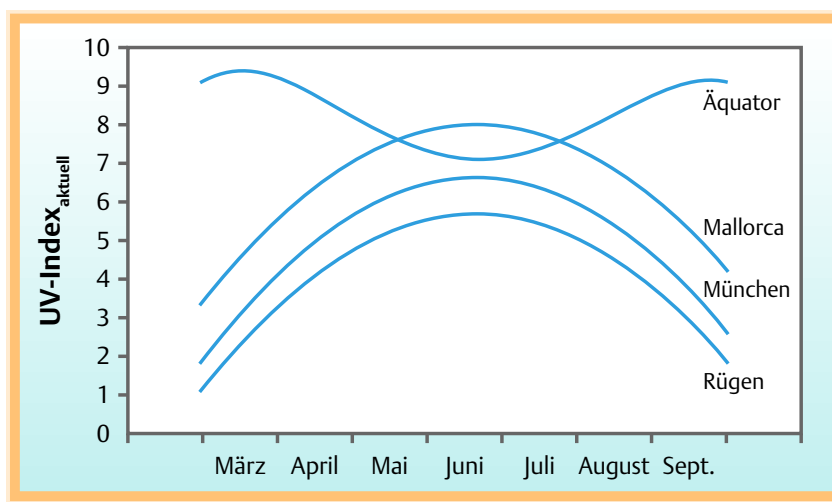

Abb. 3 UV-Index für Rügen, München, Mallorca und am Äquator (nach Engelsen [3]).

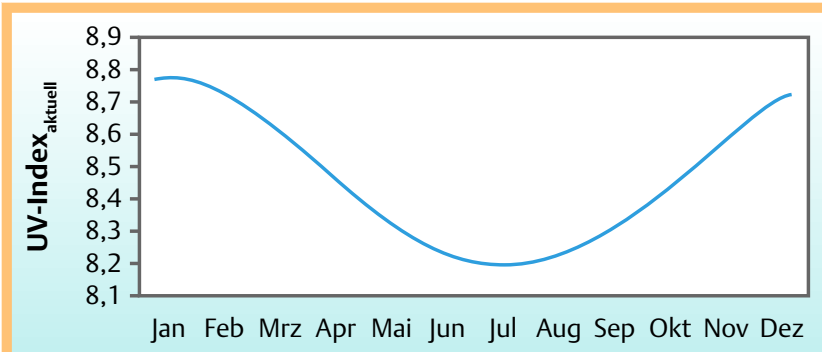

Abb.4 Einfluss der Jahreszeit auf den für Sonnenhöhenwinkel $76^{\circ}$ errechneten UV-Index.

für den vorgesehenen Zweck ausreichend, wie Vergleiche mit den Ergebnissen meteorologischer Observatorien zeigen. Der Gültigkeitsbereich des UVI wurde regional eingeschränkt, um die Anzahl der zu berücksichtigenden Randbedingungen klein zu halten. In unserem Falle ist er auf Breitenlagen wie Mitteleuropa inklusive dessen Mittelgebirge begrenzt. Auch der in den Medien angegebene, ganzzahlig gerundete UVI ist ein nur grobes Maß.

Das hier erstmals vorgestellte Verfahren ist einfach, kostenfrei und überall ohne technische Hilfsmittel anwendbar. Die zugehörigen Empfehlungen gelten für Hauttyp II. Die abgeschätzten aktuellen UVI werden im Gültigkeitsbereich durch Tages- oder Jahreszeit (siehe $\bullet$ Abb. 3) oder geografische Faktoren wie Lage und Höhe nur wenig modifiziert. Der geringe Einfluss der Jahreszeit geht aus $\bullet$ Abb. 4 hervor. 
Die individuelle Sonnen-UV-Tagesdosis hängt primär von folgenden Faktoren ab:

- von der maximalen Sonnenhöhe,

- von der Tageslänge (Jahreszeit und geografische Breite),

vom Wetter und

v von der im Freien verbrachten Zeit und der Art und Weise, wie diese verbracht wird [10].

Die Sonnenhöhe wird mit dem Sonnenhöhenwinkel $\gamma$ erfasst. Die Tageslänge geht in die Berechnung des UVI nicht ein. Das Wetter, insbesondere Bewölkung, Ozongehalt der Atmosphäre, Aerosole und Luftverunreinigung kann die Stärke des auf der Erdoberfläche auftreffenden Sonnen-UV vermindern. So wird das Verfahren eher zu große UVI ergeben.

Von besonderer praktischer Bedeutung sind die den Sonnenhöhenwinkeln von $45^{\circ}$ bzw. $36,9^{\circ}$ entsprechenden UVI (siehe - Tab. 1). Bei Sonnenhöhenwinkel $\gamma=45^{\circ}$ ist der Schatten eines aufrecht stehenden Menschen so lang wie dieser Mensch groß ist. Praktisch bedeutet das:

- Ist der eigene Schatten so lang wie man selbst groß ist, so entspricht das einem aktuellen UVI von knapp 4.

- Ein UVI von 4 entspricht einer Bestrahlungsstärke von $100 \mathrm{~mW} / \mathrm{m}^{2}$, d. h. die Sonnenbrandschwellendosis eines nicht UV-adaptierten Menschen vom Hauttyp II wäre am Mittag in Berlin unter Umständen bereits innerhalb von 42 Minuten erreichbar.

- Dann sind Schutzmaßnahmen oder eine Begrenzung der Aufenthaltsdauer erforderlich. Dies ist noch dringlicher am Vormittag, wenn die Sonne ihren Tageshöchststand noch nicht erreicht hat.

- Ist der eigene Schatten um die Mittagszeit um $1 / 3$ länger als man selbst groß ist, d. h. das Verhältnis Körpergröße/Schattenlänge ist wie $0,75 / 1,00$, so ergibt sich ein aktueller UVIndex von $<3$.

- Den aktuellen Empfehlungen der WHO und anderer Gremien zufolge sind Schutzmaßnahmen erst ab UV-Index = 3 erforderlich.

Im Extremfall kann sich ein nicht UV-adaptierter Mensch vom Hauttyp II bei UVI < 3 beispielsweise um die Mittagszeit in Berlin bereits innerhalb von 56 Minuten einen leichten Sonnenbrand zuziehen. Das zeigt, dass auch die erwähnten Empfehlungen der WHO und anderer Gremien zumindest teilweise auf einem Kompromiss beruhen, der vermutlich die bislang ausgeklammerte UV-Adaptation der Haut berücksichtigt. Streng genommen betreffen die Empfehlungen zur Begrenzung der Aufenthaltsdauer im Sonnenschein nur die nach wochenlanger UV-Karenz nicht UV-adaptierte Haut. In den zurückliegenden Jahren waren es in erster Linie australische Fachleute, die über die Gefahren übermäßiger Besonnung aufklärten und erfolgreiche Präventionskampagnen starteten. Unter dem Eindruck neuerer Erkenntnisse über Art und Ausmaß des weit verbreiteten VitaminD-Mangels sind es wiederum australische Gremien (Cancer Council Australia, Osteoporosis Australia, Australasian College of Dermatologists, Australian and New Zealand Bone and Mineral Society - New guidelines in relation to the risks and benefits of sun exposure), die jetzt zu ausreichender Besonnung aufrufen [11]. Das hier vorgestellte Verfahren zur Abschätzung des UVI empfiehlt sich als einfache Methode zur Begrenzung der täglichen Sonnen-UV-Exposition. Zudem vermag es das Verständnis darüber verbessern, zu welchen Tages- und Jahreszeiten UV-Schutzmaßnahmen sinnvoll sind oder auch nicht. Selbstverständlich kann das Verfahren für viele andere Zwecke erforderliche Präzisionsmessungen nicht ersetzen. Es sollte durch den direkten Vergleich gemessener und abgeschätzter UVI weiter überprüft werden. Möglicherweise wird es sich auch zur Kontrolle einer noch zu definierenden minimal erforderlichen UV-Tagesdosis eignen.

\section{Danksagung \\ $\nabla$}

Wir danken Herrn Dr. rer. nat. Helmut Piazena für kritische und anregende Diskussion.

\section{Abstract}

\section{A Simple Procedure for Estimating UV Index $\nabla$}

Description of a simple procedure for estimating current solar erythema-efficient ultraviolet fluence without any UV measuring device. This way the strength of sunshine can be estimated for many purposes sufficient precisely, everywhere and at any time. The procedure is based on a connection of UV Index and shadow rule, and a computer program published by O. Engelsen [3]. Only the own body-height and the length of the corresponding shadow are needed to be measured. Out of these the current UV Index can be calculated easily.

If ones shadow is

- as long as the person is tall, so the UV Index equals 4

- by $1 / 3$ longer as this person is tall, the UV Index is $<3$.

Following the current recommendations of WHO and other health institutions, protective measures are needed from UV in$\operatorname{dex}=3$ on. Constraints, advantages and limitations of the procedure are discussed. Comparisons with measured UVI are carried out.

\section{Literatur}

1 Reichrath J. The challenge resulting from positive and negative effects of sunlight: how much solar UV exposure is appropriate to balance between risks of vitamin D deficiency and skin cancer? Progr Biophys Mol Biol 2006; 92: 9-16

2 Hintzpeter B, Mensink GB, Thierfelder W, Müller MJ, Scheidt-Nave C. Vitamin D and health status correlates among German adults. European J Clin Nutr advance online publication 30 May 2007

3 http://nadir.nilu.no/ olaeng/fastrt/VitD.html

4 DIN 67501: Experimentelle Bewertung des Erythemschutzes von externen Sonnenschutzmitteln für die menschliche Haut. Berlin: Beuth Verlag, 1994

5 http://www.bfs.de/en/uv/uv2/uvi/messnetz.html

6 Holloway L. Atmospheric sun protection factor on clear days: Its observed dependence on solar zenith angle and its relevance to the shadow rule for sun protection. Photochem Photobiol 1992; 56: 229-234

7 Holloway L. Shadow rule for sun protection. J Am Acad Dermatol 1994; 31: $517-0$

8 Sliney $D H, S$. Wengraitis. Is a differenciated advice by season and region necessary? Progr Biophys Mol Biol 2006; 92: 150-160

9 Thieden F, Philipsen PA, Wulf HC. Ultraviolet radiation exposure pattern in winter compared with summer based on time-stamped personal dosimeter readings. Br J Dermatol 2006; 154: 133-138

10 Diffey $B$. A contemporary strategy for sun exposure. Expert Rev Dermatol 2; 2007: 139-142

11 http://www.cancer.org.au/documents/RisksBenefitsSunExposure03 May07.pdf 